\title{
A prevalência da tuberculose pulmonar na população privada de liberdade da central de triagem penitenciária de Ananindeua - Pará, Brasil
}

The prevalence of pulmonary tuberculosis in the population deprived of freedom from Ananindeua's penitentiary screening center, Pará, Brazil

La prevalencia de la tuberculosis pulmonar em la población privada de libertat del centro de cribado peninteciario de Ananindeua - Pará, Brasil

Josinete da Conceição Barros do Carmo ${ }^{1 *}$, Claudia Rodrigues Costa ${ }^{1}$, Luana Lima Paes ${ }^{1}$, Raphael Resende Gustavo Galvão ${ }^{1}$, Kewinny Beltrão Tavares ${ }^{1}$, Morgana da Silva Ramos¹, Carolina de Oliveira Vieira Paiva1', Laiane de Jesus Silva Viegas ${ }^{1}$, Eliane Berenice da Silva Castilho', Maria Goreti Soares Ferreira1, Rayssa Braun Imbiriba², Margareth Maria Braun Guimarães Imbiriba1.

\section{RESUMO}

Objetivo: Analisar a prevalência de tuberculose pulmonar na população privada de liberdade, encontrados na Central de Triagem Penitenciária de Ananindeua - Pará, Brasil. Métodos: Trata-se de um estudo descritivo, epidemiológico, retrospectivo com abordagem quantitativa. A pesquisa foi realizada na central de triagem Penitenciária de Ananindeua localizada na Cidade Nova IV-WE, foram analisados prontuários dos privados de liberdade que tiveram o diagnóstico de Tuberculose Pulmonar confirmado com exames laboratoriais. A pesquisa teve como amostra 30 prontuários. Resultados: $O$ estudo mostrou que os novos casos de Tuberculose Pulmonar vêm se tornando mais frequente, dos 30 prontuários, 21 são novos casos, sendo 7 de retratamento. $O$ estudo apontou que 27 deles apresentaram baciloscopia positiva $(++)$ e 3 deles com baciloscópia positiva (+++). Conclusão: Apesar dos avanços terapêuticos a tuberculose pulmonar em presídios continua prevalente e sendo um grave problema de saúde pública. Ressalta-se a escassez de estudo sobre a problemática em relação à população privada de liberdade.

Palavras-chave: Tuberculose pulmonar, População privada de liberdade, Prevalência.

\begin{abstract}
Objective: Analyze the prevalence of Pulmonary Tuberculosis in population deprived of freedom found at the Ananindeua's Penitentiary Screening Center, Pará, Brazil. Methods: This is a descriptive, epidemiological, retrospective study with a quantitative approach. The research was conducted at the Ananindeua's Penitentiary Screening Center located in Cidade Nova IV-WE, the analyzed data collection was on May 2018, in the morning, from $08 \mathrm{~h} 00$ to $12 \mathrm{~h} 00 \mathrm{~min}$ for seven days from the medical records of population in custody who had been confirmed with diagnosed Pulmonary Tuberculosis by laboratory tests. The research had as sample 30 medical records. Results: The study showed that new cases of Pulmonary Tuberculosis are becoming more frequent, from the 30 records, 21 were new cases; and 7 were retreatment. The study showed that 27 of them dah positive bacilloscopy $(++)$ and 3 had positive bacilloscopy (+++). Conclusion: Despite the therapeutic advances in Pulmonary Tuberculosis in prisons, it remains prevalent and is a serious public health issue. It is noteworthy the lack of study on the matter in relation to the population deprived of freedom.
\end{abstract}

Key words: Tuberculosis, population deprived of freedom, Prevalence rate.

1 Universidade da Amazônia (UNAMA), Belém - PA. *E-mail: enf.josineteb@outlook.com

2 Secretaria de Estado de Saúde Pública (SESPA), Belém - PA.

SUBMETIDO EM: 11/2019

ACEITO EM: 12/2019

PUBLICADO EM: $3 / 2020$

REAS/EJCH | Vol.Sup.n.42| e2497 | DOI: https://doi.org/10.25248/reas.e2497.2020 Página 1 de 9 


\section{RESUMEN}

Objetivo: Analizar la prevalencia de Tuberculosis Pulmonar en la población privada de libertad, encontrada en el Centro de Cribado Penitenciario Ananindeua - Pará, Brasil. Métodos: Estudio descriptivo, epidemiológico, retrospectivo con enfoque cuantitativo. La investigación se realizó en el Centro de Cribado Penitenciario Ananindeua ubicado en Cidade Nova IV-WE, fueron analizados los registros médicos de las personas privadas de libertad que tenían el diagnóstico de tuberculosis pulmonar confirmado por pruebas de laboratorio. La investigación tuvo como muestra 30 registros médicos. Resultados: El estudio mostró que los nuevos casos de Tuberculosis Pulmonar son cada vez más frecuentes, de los 30 registros, 21 son casos nuevos y 7 de retratamiento. El porcentaje de detenidos en el aula, en Pará, es casi el doble que la tasa nacional del $12 \%$. El estudio mostró que 27 de ellos tenían frotis positivo (++) y 3 de ellos tenían frotis positivo $(+++)$. Conclusión: A pesar de los avances terapéuticos en la Tuberculosis Pulmonar en las cárceles, sigue siendo frecuente y es un grave problema de salud pública. Es de destacar la falta de estudio sobre el problema en relación con la población privada de libertad.

Palabras clave: Tuberculosis pulmonar; Población privada de libertad; Prevalencia.

\section{INTRODUÇÃO}

A Tuberculose (TB) é uma doença causada pela bactéria Mycobacterium Tuberculosis, onde o reservatório principal é o homem, sua principal via de transmissão é através da inalação de aerossóis oriundos das vias aéreas, expelido pela tosse, espirro e fala do indivíduo contaminado, as formas clínicas são a TB pulmonar e extrapulmonar, a TB afeta principalmente adultos, porém todas as faixas etárias podem se contaminar (WHO, 2017).

A TB é uma doença cuja maior incidência está localizada nas periferias das grandes cidades, onde há uma grande concentração populacional, poucos serviços de infraestrutura, saneamento básico inexistente ou inadequado, outros locais onde pode-se ter uma alta taxa da doença são em ambientes fechados, escuros e pouco ventilados, características comuns aos ambientes prisionais do Brasil e de alguns locais do mundo, logo pode-se dizer que a TB está associada, principalmente, às péssimas condições de vida (BRASIL, 2018).

Segundo Machado JC, et al. (2016), no Brasil, desde a abertura das primeiras penitenciarias, é possível notar que as condições de saneamento básico nestes locais são péssimas, em decorrência disso acarreta-se elevadas taxas de mortalidade dos prisioneiros, entretanto, sabe-se que a maior parte dos óbitos tem sido por TB. O predomínio global da doença entre encarcerados pode ser até 50 vezes maior do que as normalidades nacionais. No ano de 2012, o predomínio de TB em todo mundo foi estimado em 169 casos por 100.000 habitantes (VALENÇA MS, et al., 2016).

Aproximadamente 75 mil casos novos e reincidentes de TB foram notificados no Brasil no ano de 2017. A quantia equivale a aproximadamente 200 casos por dia no país (BRITO AC, et al., 2018). A TB continua sendo sério problema de saúde pública a nível global. A Organização Mundial da Saúde (OMS) relata que é doença infecciosa de agente único que mais mata, ultrapassando o vírus da imunodeficiência humana (HIV). No ano de 2016, cerca de 10,4 milhões ao redor do mundo ficaram doentes devido à doença, e cerca de 1,3 milhões de pessoas morreram em decorrência da mesma. No Brasil, em 2017, foram registrados 69.569 casos novos de TB. Nesse mesmo ano, o coeficiente de incidência foi igual a 33,5 casos/100 mil hab. No período de 2008 a 2017, esse coeficiente apresentou queda média anual de 1,6\%. Em 2017, foram registrados 13.347 casos de retratamentos no país, equivalentes a $16,1 \%$ do total de casos notificados no período (BRASIL, 2018).

Atualmente no País, ultrapassam-se o número de 840 mil de privados de liberdades, contando com menos da metade de número de vagas no sistema, são menos de 400 mil vagas disponíveis, como consequência a superlotação leva a situações insalubres e até desumanas (SENADO NOTíCIAS, 2019). De acordo com Pereira LFS, et al. (2019), a tuberculose na região norte no ano de 2010, obteve casos notificados o coeficiente de 44,5 casos/100.000 habitantes, uma taxa superior que a média das taxas do Brasil, que foi de 37,2 casos/100.000 habitantes. 
No estado do Pará a taxa de incidência da tuberculose aumentou a cada ano, onde em 2014 houve 52,5 casos/100.000 habitantes e no ano de 2017 foram notificados 58,1 casos $/ 100.000$ habitantes. A taxa de incidência da doença na capital Belém no mesmo período teve 127,5 casos/100.000 habitantes em $2014 \mathrm{e}$ no ano de 2017 de 126,7 casos/100.000 habitantes (PEREIRA LFS, et al., 2019). Levando em consideração as altas taxas de TB no estado do Pará, devem-se investigar os contatos externos de familiares e visitantes nos presídios, essas investigações são dificilmente abordados pelos profissionais da saúde, o que implica em uma proliferação não só da tuberculose, mas também de outras doenças, por isso é importante que haja uma vistoria mais rigorosa com as pessoas que vão visitar esses detentos, não só por questões de segurança, mas também pela saúde para que não haja uma proliferação de doenças principalmente da TB, pois a falta de vistoria e de cuidado aumentará disseminação desacelerada da doença (CAMACHO LAB, 2015).

Segundo um estudo feito por Brito AC, et al. (2016), além de situações ambientais precárias e outros fatores condicionantes para o contagio e transmissão da TB, pode-se mencionar a falta da organização de equipes mínimas de saúde, principalmente em relação a capacitação e/ou ausência do profissional médico nestas instituições. Para Santos MNA e Sá AMM (2014), geralmente os ambientes carcerários possuem as mesmas particularidades, onde se podem citar as péssimas condições de confinamento, a superlotação que há dentro das celas, precariedade de ventilação e carência de iluminação natural. Ademais, ainda existe a questão da violência, das péssimas condições de insalubridade ambiental e o custo ao acesso a serviços de saúde dentro das prisões, tais características refletem um ambiente propicio ao aparecimento e transmissão de varias doenças, dentre as quais de forma significativa está a tuberculose.

Diante desse quadro que demonstra um elevado risco de tuberculose pulmonar em presídios, o objetivo do estudo é de analisar a prevalência de tuberculose pulmonar na população privados de liberdade, encontrado na Central de Triagem Penitenciária de Ananindeua - Pará, Brasil.

\section{MÉTODOS}

Trata-se de um estudo descritivo, epidemiológico, retrospectivo com abordagem quantitativa. A pesquisa foi realizada na Central de Triagem Penitenciária da Cidade Nova (CTCN) da região metropolitana de Belém do Pará, localizada em Ananindeua na Cidade Nova IV-WE. A central dispõe de dois técnicos de enfermagem, um enfermeiro para cada turno e dois médicos que fazem somente um atendimento por semana, cada um. A coleta de dados ocorreu no mês de maio de 2018 , no período da manhã, no horário de 08h00min as 12h00min durante 7 dias.

Após o parecer favorável da Direção da central de triagem da cidade nova - criminal, a pesquisa foi submetida para aprovação do comitê de ética da Universidade da Amazônia, № de aprovação № 88850018.9.0000.5173, atendendo as exigências do Termo de Compromisso para Utilização de Dados de Arquivo, cumprindo os requisitos da resolução 466/12 do conselho nacional de saúde, a qual incorpora sob a ótica do indivíduo e da coletividade, os quatro princípios básicos da bioética: autonomia, não maleficência, beneficência, e justiça; assegurando desta forma, o total sigilo e anonimato de todas as pessoas envolvidas neste estudo.

Os prontuários foram selecionados após um prévio contrato assinado previamente o termo de Utilização de dados. Após este contrato a pesquisa foi garantida o anonimato, através da codificação de frequências numérica para cada prontuário. $O$ instrumento utilizado para a coleta de dados um formulário previamente estruturado preparado com perguntas fechadas sobre o perfil socioeconômico e dos dados completares dos casos com Tuberculose, cujo objetivo principal foi de verificar qual a prevalência de Tuberculose Pulmonar em privados de liberdade do centro de triagem da Cidade Nova. Os dados foram analisados a partir da utilização o programa Microsoft Excel 2013, para o ordenamento dos dados que foram coletados. A partir do ordenamento elaborado e da utilização dos filtros proceder-se-á as análises com as informações sendo posteriormente exportadas para o programa Microsoft Word 2013.

A amostra foi composta por 30 prontuários dos privados de liberdade que tiveram o diagnóstico de Tuberculose Pulmonar confirmado com exames laboratoriais. Incluem-se na pesquisa os prontuários de 
homens maiores 18 anos sem limite de idade superior com diagnóstico positivo para tuberculose pulmonar, atendidos e cadastrados na central de triagem da cidade nova no período de 2012 a 2017. Como critério de exclusão foi eliminado os prontuários de homens que não estavam na faixa etária estabelecida bem como prontuários de homens que não foram atendidos e cadastrados na central de triagem da cidade nova, e os nãos portadores de tuberculose.

\section{RESULTADO E DISCUSSÃO}

O estudo obteve de 30 portuários da população privada de liberdade da central de triagem penitenciária da cidade nova infectados com tuberculose. A Tabela 1 demonstra a faixa etária dos privados de liberdade da central de triagem onde observa-se que a faixa etária de maior expressão é entre os indivíduos adultos jovens, situados entre 20 a 30 anos de idade, que coincide com o padrão nacional, onde a Tuberculose Pulmonar em Pessoas Privados de Liberdade (PPL) tem maior acometimento da doença na faixa etária entre 20 e 49 anos de idade (FREITAS WMTM, et al., 2016).

Tabela 1 - Faixa etária dos privados de liberdade da central de triagem penitenciária da cidade nova, estado do Pará, 2018.

\begin{tabular}{c|c}
\hline Faixa etária & N. \\
\hline $20-30$ & 18 \\
\hline $31-40$ & 8 \\
\hline $41-50$ & 3 \\
\hline $51-60$ & 1 \\
\hline Total & 30 \\
\hline Fonte: Carmo JCB, et al., 2019. &
\end{tabular}

A Tabela 2 demonstra a cor dos privados de liberdade, onde a maioria dos indivíduos é de coloração parda, algo que não apresenta surpresas, considerando que as pessoas que se identificam como pardas compõem a maioria da população paraense segundo último levantamento realizado pelo Instituto Brasileiro de Geografia e Estatística (IBGE) em 2010, onde 69,9\% da população paraense é considerada parda, 21,6\% branca, 7\% preta, 0,9\% amarela e 0,5\% indígena (IBGE, 2010).

Tabela 2 - Cor dos privados de liberdade da central de triagem penitenciária da cidade nova, estado do Pará, 2018.

\begin{tabular}{c|c}
\hline Raça/cor & N.o \\
\hline Parda & 20 \\
\hline Branco & 4 \\
\hline Preta & 6 \\
\hline Total & 30 \\
\hline Fonte: Carmo JCB, et al., 2019. &
\end{tabular}

A Tabela 3 demonstra a escolaridade dos privados de liberdade, onde a maioria dos detentos possuem ensino fundamental, características que mostra a realidade educacional dessa população. Levando em consideração os dados obtidos neste estudo observou-se que quanto maior o nível de escolaridade menor vai sendo a quantidade de detento. 
Tabela 3 - Escolaridade dos privados de liberdade da central de triagem penitenciária da cidade nova, estado do Pará, 2018.

\begin{tabular}{c|c}
\hline Escolaridade & N.․․ \\
\hline Ensino Fundamental. & 23 \\
\hline Ensino Médio & 7 \\
\hline Superior & 0 \\
\hline Total & 30 \\
\hline Fonte: Carmo JCB, et al., 2019. &
\end{tabular}

A Tabela 4 evidencia que os detentos "sem ocupação" representam a maior quantidade em detrimentos dos "com ocupação". No que se refere a ocupação $40 \%$ dos 30 prontuários pesquisados não tinham ocupação. O desemprego no país e no estado é uma realidade enfrentada pelos brasileiros, dados confirmados pelo IBGE (IBGE, 2018).

Tabela 4 - Ocupação dos privados de liberdade da central de triagem penitenciária da cidade nova, estado do Pará, 2018.

\begin{tabular}{c|c}
\hline Ocupação & N.․ \\
\hline Sem ocupação & 19 \\
\hline Com ocupação & 11 \\
\hline Total & 30 \\
\hline Fonte: Carmo JCB, et al., 2019. &
\end{tabular}

Segundo Santos MNA e Sá AMM (2014), no ano de 2011, levando em conta uma população de privados de liberdade de 12.000 pessoas, o resultado do coeficiente de incidência da TB foi por volta de 1.150/100.000, isto é, aproximadamente 25 vezes mais elevada que a incidência entre os paraenses e em torno de 32 vezes maior que a ocorrência nacional no mesmo ano para os indivíduos em geral. No País, constata-se que nos últimos anos marcaram para uma desaceleração tanto no número de diagnostico quanto na mortalidade por TB. Mas ainda continua alta a quantidade de novos casos de TB, principalmente entre populações consideradas mais vulneráveis, tais como os indígenas, PPL, e em situação de rua (BRITO AC, 2018).

A superlotação é um fator de risco causador para os elevados índices de contatos da TB nas penitenciarias do Brasil. A população prisional é considerada mais suscetível a contrair doença. Além da superlotação, podese dizer que as situações de atendimento em saúde nas penitenciarias brasileiras são motivo de inquietação por parte de especialistas, pelo fato de ter aglomeração de indivíduos em um ambiente acarretando o contato de pacientes infectados com aqueles presidiários que não tem a doença. Portanto, o diagnóstico no início é essencial para conter o avanço da doença tanto na pessoa infectada como no ciclo de transmissão (ONU, 2016).

\section{Características clínicas dos privados de liberdade}

O resultado mostra que os novos casos de TB vêm se tornando mais frequente, dos 30 prontuários, 21 são novos casos, sendo 7 de retratamento. A Organização Mundial de Saúde (OMS) iniciou o segundo ano da campanha mundial e teve em torno de 10 milhões de novos casos notificados por ano, induzindo mais de um milhão de pessoas a óbito. Nos últimos anos, a preocupação com esses números elevados fez com que está entidade redefinisse a classificação de países prioritários para o período de 2016 a 2020 (BRASIL, 2017). 
No que se refere ao diagnóstico, dos 30 prontuários pesquisados, 30 foram diagnosticados com o exame de escarro. Sabe-se que para o controle da TB, o diagnostico precoce é um dos principais meios a serem alcançados, onde se devem levar em consideração diversos aspectos, tais como os clínicos, epidemiológicos e os laboratoriais. Quando se fala do aspecto clínico-epidemiológico dois elementos são de suma importância, a anamnese e o exame físico, onde são responsáveis para obtenção dos sinais e sintomas que indicam a doença, como as lesões na orofaringe, adenopatias periféricas, alterações ventilatórias e obstrução bronquial (FARGA V e CAMINERO JA, 2011).

Os problemas decorrentes da superpopulação carcerária é uma realidade do país. O sistema penitenciário, centrado na pena de prisão em regime fechado, uma das mais cruéis violências praticadas com aval institucional (VALENÇA MS, et al., 2016). Dentre as condições que propiciam a proliferação de epidemias e ao desenvolvimento de patologias e psicopatologias são: estruturas arquitetônicas em ruínas, superlotação úmidas e escuras, péssima alimentação, sedentarismo, uso de drogas e falta de higiene (BRASIL, 2014). Fato este constatado nesta pesquisa.

Dentre as formas da TB, a forma pulmonar é a mais importante e prevalente entre elas e se progride em um curto intervalo de tempo após a primeira exposição ou após anos da exposição, quando se manifestará após uma reativação endógena no organismo. Dentre as formas de tuberculose pulmonar têm-se a pneumonia tuberculosa, tuberculose bronquial, silício tuberculose, tuberculoma e a tuberculose de lobos inferiores (FARGA V; CAMINERO JA, 2011). A forma de tuberculose pulmonar, foi a mais prevalente é a de maior incidência no universo pesquisado. A TB na forma pulmonar, além de ser mais encontrada, é considerada a relevante para a saúde pública, pois é a forma pulmonar bacilífera, é a responsável pela manutenção da cadeia de transmissão da doença (BRASIL, 2018). Pondera que a busca ativa dos sintomáticos respiratórios é a estratégia primordial para buscar controlar a TB, permitindo a detecção precoce das formas pulmonares (SPAGNOLO LML, 2018). Os Dados relatados por esta pesquisa mostram que dos 30 detentos, todos apresentam a tuberculose pulmonar, obtendo um potencial de $100 \%$.

A pesquisa aponta que dos 30 prontuários pesquisados, 27 deles apresentam baciloscopia positiva (++) e 3 deles com baciloscópia positiva (+++), as cruzes encontradas nos diagnósticos demostram o Bacilo Álcool Ácido Resistente (BAAR) por campo observados durante a analise da baciloscopia de escarro, duas cruzes (++) representam 1 a 10 BAAR por campo em 50 campos observados e 3 cruzes (+++) representam mais de 10 BAAR por campo em 20 campos observados (BRASIL, 2019). Dentre os meios para se diagnosticar a TB nos serviços de saúde pública, a baciloscópia é o método mais utilizado, pois a mesma trata-se de uma analise rápida e de baixo custo, este procedimento busca pesquisar a presença do bacilo álcool-ácidoresistente (BAAR). Através da baciloscópia pode-se detectar cerca de $80 \%$ dos casos de TB pulmonar, no entanto o método possui a baixa sensibilidade, havendo a possibilidade de ocorrer resultados falso-negativo (BRASIL, 2019).

É recomendado que se tenha a descentralização dos serviços de diagnósticos para diversos pontos das Redes de Atenção a Saúde (RAS), não devendo apenas estarem concentrados nas unidades de saúde publica, sendo representadas pelas unidades básicas e estratégia saúde da família, mas deve-se ampliar os serviços de diagnósticos com o objetivo de alcançar os hospitais, unidades de urgência e emergência e outros pontos das RAS, mas para tal, é necessários que os gestores de saúde autorizem tal procedimento de descentralização (BRASIL, 2019).

Um ponto que deve ser discutido é a probabilidade do individuo contrair a doença a partir de uma patologia preexistente, como o HIV. Estudos relatam que a possibilidade de um indivíduo com HIV infectado pelo bacilo da TB desenvolver a doença é cerca $10 \%$ ao longo da vida, já no indivíduo infectado pelo HIV e sem intervenção terapêutica, essa probabilidade passa a ser de 10\% ao ano (MENDONSA GR, et al., 2012). Além do HIV, o álcool e o tabaco estão associados ao aumento da incidência da tuberculose, a um pior resultado do tratamento e um risco maior de recidiva após o seu término (SILVA DR, et al., 2018) Santos MNA e Sá AMM (2014), diz que todos aqueles que convivem no mesmo ambiente com indivíduos diagnosticados com TB, a avaliação para o diagnóstico deve levar em consideração o tempo de permanência com o infectado, a forma da doença e o ambiente de contato. 
Durante a pesquisa não foram encontradas notas nos prontuários dos detentos relatando sobre presença de um profissional de saúde na observação da autoadmiração dos medicamentos por parte dos encarcerados. A tuberculose é uma patologia que tem cura comprovada nos casos novos sensíveis aos fármacos. Para que haja a interrupção da cadeia de transmissão da tuberculose a principal conduta a se fazer é a busca dos pacientes bacilíferos para o início precoce do tratamento. O Ministério da saúde aconselha que o profissional de saúde, através do Tratamento Diretamente Observado (TDO), assista o doente durante a ingestão dos medicamentos (BRASIL, 2018).

Atividades de educação continuada entre os profissionais de saúde sobre a importância ao TDO se faz necessário com intuito de minimizar quaisquer problemas relacionados à saúde, seja sobre a observação do tratamento da TB ou quaisquer outras patologias. Com isso, profissionais e gestores dos presídios devem conhecer o ambiente que estão atuando e buscar avaliar os perigos que podem acontecer em relação à saúde de todos do ambiente, e a partir do reconhecimento dos riscos devem-se traçar medidas com intuito de preveni-los.

Conforme recomendações do Ministério da Saúde, a forma de tratamento deveria ser supervisionada e orientada pelo um profissional da área da saúde (BRASIL, 2018). Mas, na realidade as formas de tratamento no sistema prisional não obedecem a essas normas, conforme dados pesquisados. Quanto aos exames complementares, afirma-se que dos 30 detentos, apenas três fizeram exames de cultura de escarro e dois detentos raio-x de tórax, totalizando em 5 detentos. Os resultados demonstram a gravidade da falta de exames para esses indivíduos. Dentre as técnicas de diagnostico da doença, a cultura do bacilo da TB é um método que pode aumentar a sensibilidade diagnostica e ainda possui uma especificidade alta, o ponto negativo que esse método possui é a respeito ao resultado do teste, pois é um procedimento mais demorado (FARGA V, CAMINERO JA, 2011).

\section{CONCLUSÃO}

O estudo constatou que a população privada de liberdade, na maioria, possuía idade de 20 á 30 anos e da cor parda, a prevalência de novos casos de tuberculose e todos apresentaram tuberculose pulmonar. Assim, a pesar todos os avanços terapêuticos para o tratamento da doença ainda continua sendo um grave problema de saúde pública e a contaminação e propagação da doença merece ser mais bem observada. Acredita-se que se necessitam ações de prevenção e de vigilância epidemiológica dentro das penitenciarias e que os protocolos assistenciais sejam seguidos, e ainda que os profissionais da saúde possam estar qualificados e sensibilizados para uma questão tão importante que é o controle da tuberculose. Espera-se que o estudo contribua para uma futura assistência mais aprimorada para os detentos, observando que os fatores sociais e a ausência de políticas públicas de prevenção e tratamento têm interferido para a cura da doença.

\section{REFERÊNCIAS}

1. BRASIL. 2017. In: Tuberculose. Rio de Janeiro: Ministério da Saúde.

2. Brasil. Conselho Nacional de Justiça. 2014. In: Dados das Inspeções nos Estabelecimentos Penais.

3. BRASIL. Implantação do Plano Nacional pelo Fim da Tuberculose como Problema de Saúde Pública no Brasil: primeiros passos rumo ao alcance das metas. Brasil: Boletim epidemiológico, 2018; 11(49): 1-18.

4. BRASIL. Ministério da Saúde. Secretaria de Vigilância em Saúde. Departamento de Vigilância das Doenças Transmissíveis. Manual de Recomendações para o Controle da Tuberculose no Brasil. 2nd ed. Brasília: Ministério da Saúde; 2019; 364 P.

5. BRITO AC, et al. Análise das publicações sobre tuberculose no sistema prisional. Revista Corpus Hippocraticum, 2017; 1(1): 1-9.

6. CAMACHO LAB, et al. Análise da implantação do programa de controle da tuberculose em unidades prisionais no Brasil. Caderno de Saúde Pública, 2015; 31(3): 543-554.

7. PEREIRA LFS, et al. Epidemiologia da tuberculose no estado do Pará. Brazilian Journal of Health Review, 2019; 2 (2): 800-808. 
8. FARGA V, CAMINERO JA. Tuberculosis. 3nd ed. Santiago de Chile: Mediterrâneo, 2011; 484p.

9. FREITAS WMTM, et al. Perfil clínico-epidemiológico de pacientes portadores de tuberculose atendidos em uma unidade municipal de saúde de Belém, Estado do Pará, Brasil. Revista Pan-Amazonica de Saúde, 2016; 7(2): 4550.

10. INSTITUTO BRASILEIRO DE GEOGRAFIA E ESTATíSTICA- IBGE. 2010. In: Tabela 2094 - População residente por cor ou raça e religião. BRASIL: IBGE.

11. INSTITUTO BRASILEIRO DE GEOGRAFIA E ESTATÍSTICA- IBGE. 2018. In: Desemprego volta a crescer no primeiro trimestre de 2018. BRASIL: Estatística Sociais.

12. MACHADO JC, et al. A incidência de tuberculose nos presídios brasileiros: Revisão Sistemática. Revista de Atenção à Saúde, 2016; 14(47): 88-84.

13. MENDONÇA GR, et al. Tuberculose, HIV e pobreza: tendência temporal no Brasil, Américas e mundo. Jornal brasileiro de Pneumologia, 2012; 38(4): 511-517.

14. ORGANIZAÇÃO DAS NAÇÕES UNIDAS- ONU. Brasil. 2016. In: Tuberculose nos presídios brasileiros é emergência de saúde e de direitos humanos, dizem especialistas.

15. SANTOS MNA, Sá AMM. O ser-portador de tuberculose em prisões: um estudo de enfermagem. Escola Anna Nery, 2014; 18(2): 350-355.

16. SENADO NOTÍCIAS. 2019. In: País tem superlotação e falta de controle dos presídios. Brasil: Agencia do Senado.

17. SILVA DR, et al. Fatores de risco para tuberculose: diabetes, tabagismo, álcool e uso de outras drogas. Jornal Brasileiro de Pneumologia, 2018; 44(2): 145-152.

18. SPAGNOLO LML, et al. Detecção da tuberculose: fluxo dos sintomáticos respiratórios e resultados alcançados. Revista Brasileira de Enfermagem, 2018; 71(5): 2543-2551.

19. VALENÇA MS, et al. Tuberculose em presídios brasileiros: uma revisão integrativa da literatura. Revista Ciência e Saúde Coletiva, 2016; 21(7): 2147-2160.

20. WORLD HEALTH ORGANIZATION (WHO). 2017. In: Global tuberculosis report. Geneva: World Health Organization; 2017. 